\title{
THE EXCESS SECULAR CHANGE IN THE OBLIQUITY OF THE ECLIPTIC AND ITS RELATION TO THE INTERNAL MOTION OF THE EARTH
}

\author{
CHUICHI KAKUTA \\ International Latitude Observatory, Mizusawa, Iwate \\ and \\ SHINKO AOKI \\ Tokyo Astronomical Observatory, Mitaka, Tokyo
}

\begin{abstract}
The previous result (Aoki, 1969) on the explanation of the excess secular change in the obliquity of the ecliptic frictional couplings in the rigid constituents, the mantle and the core, is extended by using a model of an elastic and electrically conducting mantle and a hydromagnetic core. The secular change of the obliquity of the ecliptic referred to the mantle is found to be $1 / 3.2$ times of the observed value, if the electrical conductivities of the fluid core and the mantle are assumed to be $3 \cdot 10^{-6} \mathrm{emu}$ and $3 \cdot 10^{-9} \mathrm{emu}$ respectively. A large secular deceleration of the Earth's rotational speed obtained in the previous result is proved to be strongly reduced because of weak excitation of the perturbing potential for a long time variation.
\end{abstract}

\section{Introduction}

Aoki (1969: hereafter referred to as paper I) explained the excess secular change in the obliquity of the ecliptic referred to the mantle by a frictional coupling between the rigid constituents, the mantle and the core, of which the axes of figures do not coincide. Non-alignment of the figure axes of the mantle and the core is essential to cause a component of the precessional torque along the axes directed to the Moon and the Sun, and brings about the excess in the obliquity of the mantle. Frictional coupling near the top layer of the fluid core due to a large velocity shear maintaines nonalignment of the axes of the mantle and the core. In paper I the frictional coupling is assumed to be proportional to the differential angular velocity between the mantle and the core, and unfortunately Aoki found a discrepancy between theory and observation for the gradual change of the rotational speed when he used the result deduced from the obliquity. Aoki and Kakuta (1971: herafter referred to as paper II) attempted to modify the model of the Earth by the introduction of a model of the elastic mantle and the viscous fluid core.

The aim of this study is to extend our arguments made in paper II about the excess secular change of the obliquity to the mantle, taking into consideration the magnetic coupling between the elastic and electrically conducting mantle and the hydromagnetic core. Electrical conductivity of both the mantle and the fluid core is assumed to be finite so as to induce a magnetic torque between the mantle and the core. The viscous coupling is essential to induce non-alignment of the axes of the mantle and the core; on the contrary, the internal motion of the fluid core itself may be treated as that of a frictionless fluid, this has been studied well by the recent theory of Earth tides (Jeffreys 
and Vicente, 1957; Molodensky, 1961). The hydromagnetic motion in the finite conducting core transfers the tidal energy into the conducting mantle along the lines of force of the main poloidal field and is dissipated there. The magnetic coupling between the mantle and the core may be caused by the magnetic stress at the core-mantle boundary (Rochester and Smylie, 1965; Rochester, 1968; Hide, 1969; Stacey, 1970). As the magnetic stress in the lower conducting mantle is more dissipative than in the core, the motion of the mantle will cause a phase lag relative to the tidal potential. The phase lag results in the non-alignment of the figure axis of the mantle to that of the fluid core. This may coincide with the idea of the non-alignment of the axes of figures of the mantle and the core of paper $I$.

The frictional layer between the rigid constituents in Paper I can be extended to the magnetic viscous layer in the hydromagnetic viscous core. In the uniform rotating earth's frame the precessional motion is diurnal but the differential rotation is of a slow motion. The skin depth for each phenomenon depends on the frequency of disturbances relative to the rotating frame. The isotropic frictional coupling in paper $I$ is, therefore, modified to be anisotropic.

The tangential stress due to the hydromagnetic motion at the mantle-core boundary may induce a torsion, a rotational component of displacement relative to the hydrostatic equilibrium state, in the elastic mantle in addition to two modes of displacement associated with changes of the potential (Takeuchi, 1950). This gives also a differential motion of the mantle relative to the uniform rotating frame. Some modes of the rotational component may be coupled with the tidal potential of the spherical harmonics of the second order and give a rigid rotation of the mantle relative to the rotating frame.

\section{A Model of the Earth}

The reference frame is taken to coincide with the mean figure axes. We assume that the rotational vector of the reference frame also directs to, say, the $Z$-axis of the reference frame in the mean for a long interval of time. The main magnetic field is assumed to be uniform and parallel to the $Z$-axis. The torsional displacement due to the tangential component of the magnetic stress at the mantle-core boundary is expressed by the rotational component of displacement derived by Takeuchi (1950). A model of the Earth is assumed to consist of the homogeneous elastic mantle having a finite electrical conductivity and the fluid core.

\section{Equations of Motion in the Rotating Frame}

Equations of motion of the deformable Earth are formulated by considering the effect of the temperature fluctuations and the magnetic field on the fluid core (Molodensky, 1961). The induced potential caused by the redistribution of mass due to the tidal potential satisfies the Poisson equation. Motions in the rotating fluid core as well as in the elastic mantle are expressed in terms of the perturbing potential, the excess of the pressure per unit mass to the additional potential, defined by Molodensky (1961). 


\section{Equations of Rotational Motion}

Torque and additional relative angular momentum due to the rotational motion of the mantle and the fluid core referring to the rotating frame written by the perturbing potential, and finally by the tidal disturbing functions. Torque acting on the whole Earth consists of the precessional torque, as if the Earth were a spheroidal rigid, and the coupling torque due to the interaction between the hydromagnetic fluid core and the elastic and the conducting mantle. The total sum of the tangential component of stress at the mantle-core boundary tends to zero. The coupling torque, therefore, is determined by the elastic stress on the surface of the mantle. Contributions to relative angular momentum are due to the relative motion in the rotating frame and to the change of the moment of inertia. Finally we obtain equations of the rotational motion of the Earth including the mantle and the core. The angular velocity of the rotating system to the fixed axis in space are expressed in terms of the Eulerian angles, which are shown in paper $I$.

\section{Conclusion}

The results are obtained by assuming the initial condition, coincidence of axis of rotation with that of the reference frame, as follows:

(1) The magnetic energy dissipation in the mantle caused a phase lag in the motion of the mantle. It is about $3 \cdot 10^{-7} \mathrm{rad}$. A part of the precessional torque may be formed on the axis which directs to the Moon and the Sun. This torque may change the obliquity of the ecliptic of the mantle, not the whole Earth. If the electrical conductivity of the fluid core and of the mantle are assumed to be $3 \cdot 10^{-6} \mathrm{emu}$ and $3 \cdot 10^{-9} \mathrm{emu}$ respectively, the secular change of the obliquity of the ecliptic to the mantle is amount of $1 / 3.2$ compared with the observed value. This argument is comparable with the results of paper I.

(2) Variations of the Earth's rotational speed, as far as the precessional motion is concerned, are slow. Mutual coupling between the elastic mantle and the hydromagnetic core decreases rapidly with the factor of the square of the angular frequency of the variation referred to the rotating frame. The rate of the deceleration of the Earth's rotation obtained in paper I may be strongly reduced.

\section{References}

Aoki, S.: 1969, Astron. J. 74, 284.

Aoki, S. and Kakuta, C.: 1971, Celest. Mech.4, 171.

Hide, R.: 1969, Nature 222, 1055.

Jeffreys, H. and Vicente, R. O.: 1957, Monthly Notices Roy. Astron. Soc. 117, 142.

Molodensky, S.: 1961, Comm. Obs. Roy. Belgique, Sér. Géophys. 58, 25.

Rochester, M. G. and Smylie, D. E.: 1965, Geophys. J. 10, 289.

Rochester, M. G.: 1968, J. Geomagn. Geoelec. 20, 387.

Stacey, F. V.: 1970, in L. Mansinha et al. (eds.), Earthquake Displacement Fields and the Rotation of the Earth, D. Reidel Publ. Co., Dordrecht, Holland, p. 176.

Takeuchi, H.: 1950, Trans. Am. Geophys. Un. 31, 651. 


\section{DISCUSSION}

W. Fricke: As far as observational evidence is concerned, the possibility of a rotation of the Earth's mantle about an axis through the equinox cannot be excluded. If you are satisfied with an angular velocity of about 0 !1 per century, no objection could be made on the basis of available observations.

$S$. Aoki: I think there might be a controversy concerning the calculated value here compared with the observed value: our calculation gives us $\frac{1}{3}$ of the observed value only. But there are problems on both sides, observational value has a difficulty which Prof. Fricke has shown. On the other hand, the calculated value has its own problem on the model used. These are both big problems. For this reason I do not care on the discrepancy so far.

S. Debarbat: Is the model you have been speaking of the same one as your published in the paper 'The Effect of a Compressible Core on Molodensky's Theory of Earth Tides' in Publ. Astron. Soc. Japan 22, No. 2, 1970?

C. Kakuta: No, it is not the same one. This model is based on the viscous coupling at the mantle-core boundary. 